\title{
A Robust Asynchronous Multiuser STBC-OFDM Transmission Scheme for Frequency-Selective Channels
}

\author{
Dandan Wang, Student Member, IEEE, Hlaing Minn, Senior Member, IEEE, and Naofal Al-Dhahir, Fellow, IEEE
}

\begin{abstract}
In this paper, we propose a robust STBC transmission scheme to combat timing synchronization errors over frequency-selective multiple-access channels. We start by deriving the equivalent channel model in the presence of timing synchronization errors. Based on this correlated equivalent channel model, the statistical channel power gain profile is analyzed and shown to exhibit unequal channel power gains on different subcarriers. Furthermore, a robust statistical bit loading algorithm is proposed to optimize the BER performance in scenarios where link adaptation based on instantaneous channel information is infeasible or undesirable. Simulation results show that our proposed scheme is robust to timing synchronization errors, providing around $7.5 \mathrm{~dB}$ SNR advantage at the BER of $10^{-4}$ over the conventional scheme in various channel environments.
\end{abstract}

Index Terms-STBC, OFDM, bit loading, asynchronous, multiple-access, ISI.

\section{INTRODUCTION}

$\mathbf{M}$ ULTIPLE-INPUT-MULTIPLE-OUTPUT (MIMO) systems can utilize the spatial diversity and multiplexing gains using spatially-separated antennas in a multipath scattering environment. In broadband systems, space-time block codes (STBC) have been integrated with orthogonal frequency division multiplexing (OFDM) by implementing STBC at a block level in a structure commonly known as ST-OFDM [1]. Since wireless communications is challenged by limited spectral resources, multi-user spacial multiplexing has recently received considerable attention. Multi-user MIMO systems can significantly improve system throughput via transceiver signal processing if the number of transmit antennas is much larger than the number of receive antennas [2]. In this context, a space-time transceiver was investigated in [3] over flatfading channels assuming two perfectly-time-synchronized users. However, in practical systems, it is very difficult, if not impossible, to achieve perfect synchronization among all uplink users. In [4], the authors considered an equivalent channel model for a flat-fading system with synchronization errors in a cooperative network. Since the channels are usually frequencyselective in broadband systems, this motivates us to investigate the multi-user MIMO transceiver with synchronization errors

Manuscript received May 31, 2007; revised October 30, 2007 and March 26, 2008; accepted June 10, 2008. The associate editor coordinating the review of this letter and approving it for publication was A. Chockalingam. This work was supported in parts by the Erik Jonsson School Research Excellence Initiative, the University of Texas at Dallas, USA, the Texas Advanced Technology Program (ATP) under Contract 009741-0023-2003, the National Science Foundation (NSF) under Contracts CCF 04-30654 and DMS 05-28010, and a gift from Texas Instruments Inc. The material in this paper was presented in part at the IEEE Sarnoff Symposium, Princeton, NJ, USA, May 2007.

The authors are with the Dept. of Electrical Engineering, University of Texas at Dallas, Richardson, TX 75083 (e-mail: \{dxw053000, hlaing.minn, aldhahir\}@utdallas.edu).

Digital Object Identifier 10.1109/T-WC.2008.070577 over frequency-selective channels in spacially-multiplexed STOFDM systems.

By exploiting the special structure of the statistical channel profile of the equivalent channel model in the presence of timing synchronization errors, we propose a robust transmission scheme to combat the synchronization errors. The proposed scheme is appealing to scenarios where link adaptation based on instantaneous channel information is infeasible or undesirable.

This paper is organized as follows. In Section II, the system set-up and the equivalent channel model with synchronization errors are presented. The statistical power profile of the equivalent channel model and a robust statistical bit loading algorithm are presented in Section III. In Section IV, simulation results are presented, and conclusions are drawn in Section V.

\section{System Model}

\section{A. System Setup}

In this paper, we consider an uplink system in which two users access the base station at the same time within the same bandwidth using spatial division multiple access (SDMA). Each user has $N_{t}$ transmit antennas, and there are $N_{r}$ receive antennas at the base station. Each user implements orthogonal space-time block codes (OSTBC) [5] on each subcarrier over adjacent OFDM symbols each consisting of $N$ sub-carriers. At the base station, the FFT is first applied and then an interference cancelation scheme is employed in the frequency domain on each sub-carrier to decode the independent information streams from different users. In this paper, we assume that all the receive antennas at the base station operate with the same clock and transmissions on all the transmit antennas at each user are also synchronous. In addition, the arrival delays from different transmit antennas of the same user are assumed to be the same. However, the arrival delays can vary from user to user. Then, the base station has to decide on a single sampling time reference point for the two users with different arrival delays, which induces a timing synchronization error for each user.

\section{B. Equivalent Channel Model}

In this subsection, we will derive the equivalent multipath channel model with synchronization errors. The distributions of the channel taps between different transmit and receive antenna pairs are assumed to be the same. Without loss of generality, in the following, we analyze the equivalent channel between the $j$ th antenna of the $i$ th user and the first receive 
antenna. The original channel between the $j$ th antenna of the $i$ th user and the first receive antenna is

$$
g_{i, j}(t)=\sum_{l=1}^{L_{i, j}} g_{i, j, l} \delta\left(t-t_{i, j, l}\right)
$$

where $L_{i, j}$ is the number of channel taps, $g_{i, j, l}$ and $t_{i, j, l}$ are the channel tap gain and the delay of the $l$ th channel tap, respectively. For notation simplicity, we neglect the subscripts $\{i, j\}$ in the following. Denote the combined transmit and receive pulse shaping filter as $p(t)$. Let $\tau$ denote the timing error with respect to the ideal samples for the $n_{i, j}$ th symbol of the $j$-th antenna of the $i$-th user. Then, the equivalent channel is given by

$$
h_{a}(t)=g(t) \otimes p(t+\tau)=\sum_{l=1}^{L} g_{l} p\left(t+\tau-t_{l}\right),
$$

where $\otimes$ denotes the linear convolution operation.

The equivalent discrete-time channel is

$$
h_{a}\left(n T_{s}\right)=\sum_{l=1}^{L} g_{l} p\left(n T_{s}+\tau-t_{l}\right),
$$

where $\frac{1}{T_{s}}$ is $N$ times the sub-carrier spacing. The equivalent channel vector is defined as $\boldsymbol{h}=\left[\cdots, h_{a}\left(-2 T_{s}\right) h_{a}\left(-T_{s}\right) h_{a}(0) h_{a}\left(T_{s}\right) h_{a}\left(2 T_{s}\right) \cdots\right]^{T}$.

\section{Special Case:}

Let $p(t)$ be the raised cosine pulse shape function given as

$$
p(t)=\frac{\sin \left(\pi t / T_{s}\right)}{\pi t / T_{s}} \frac{\cos \left(\alpha \pi t / T_{s}\right)}{1-4 \alpha^{2} t^{2} / T_{s}^{2}},
$$

where $\alpha$ is the roll-off factor. For the truncated pulse shape function with the first-order side lobes only, $p(t)$ is nonzero only within $-2 T_{s} \leq t \leq 2 T_{s}$.

For $0<\tau<T_{s}$, and $t_{l}=(l-1) T_{s}$, where $l \geq 1$ is an integer, define $p_{0}=p\left(\tau-T_{s}\right), p_{1}=p(\tau), p_{2}=p\left(\tau+T_{s}\right)$ and $p_{3}=p\left(\tau-2 T_{s}\right)$. Then, the equivalent channel vector can be constructed from the original channel taps as

$$
\boldsymbol{h}=\boldsymbol{P} \boldsymbol{g}
$$

where

$$
\boldsymbol{P}=\left(\begin{array}{ccccccc}
p_{3} & 0 & 0 & 0 & \ldots & 0 & 0 \\
p_{0} & p_{3} & 0 & 0 & \ldots & 0 & 0 \\
p_{1} & p_{0} & p_{3} & 0 & \ldots & 0 & 0 \\
p_{2} & p_{1} & p_{0} & p_{3} & \ldots & 0 & 0 \\
0 & p_{2} & p_{1} & p_{0} & \ldots & 0 & 0 \\
. & . & . & . & \ldots & . & . \\
0 & 0 & 0 & 0 & \ldots & p_{0} & p_{3} \\
0 & 0 & 0 & 0 & \ldots & p_{1} & p_{0} \\
0 & 0 & 0 & 0 & \ldots & p_{2} & p_{1} \\
0 & 0 & 0 & 0 & \ldots & 0 & p_{2}
\end{array}\right) \text { and } \boldsymbol{g}=\left(\begin{array}{c}
g_{1} \\
g_{2} \\
g_{3} \\
g_{4} \\
\vdots \\
g_{L}
\end{array}\right) .
$$

For $-T_{s}<\tau<0$, define $p_{0}^{\prime}=p\left(\tau-T_{s}\right), p_{1}^{\prime}=p(\tau)$, $p_{2}^{\prime}=p\left(\tau+T_{s}\right)$ and $p_{3}^{\prime}=p\left(\tau+2 T_{s}\right)$. Then, the equivalent channel vector is given by

$$
\boldsymbol{h}=\boldsymbol{P}^{\prime} \boldsymbol{g},
$$

where

$$
\boldsymbol{P}^{\prime}=\left(\begin{array}{ccccccc}
p_{0}^{\prime} & 0 & 0 & 0 & \ldots & 0 & 0 \\
p_{1}^{\prime} & p_{0}^{\prime} & 0 & 0 & \ldots & 0 & 0 \\
p_{2}^{\prime} & p_{1}^{\prime} & p_{0}^{\prime} & 0 & \ldots & 0 & 0 \\
p_{3}^{\prime} & p_{2}^{\prime} & p_{1}^{\prime} & p_{0}^{\prime} & \ldots & 0 & 0 \\
0 & p_{3}^{\prime} & p_{2}^{\prime} & p_{1}^{\prime} & \ldots & 0 & 0 \\
. & . & . & . & \ldots & . & . \\
0 & 0 & 0 & 0 & \ldots & p_{1}^{\prime} & p_{0}^{\prime} \\
0 & 0 & 0 & 0 & \ldots & p_{2}^{\prime} & p_{1}^{\prime} \\
0 & 0 & 0 & 0 & \ldots & p_{3}^{\prime} & P_{2}^{\prime} \\
0 & 0 & 0 & 0 & \ldots & 0 & p_{3}^{\prime}
\end{array}\right)
$$

\section{Robust Statistical Bit Loading Scheme}

In this section, we first analyze the statistical power profile of the equivalent channel model and then design a statistical bit loading algorithm which utilizes this channel power profile knowledge.

\section{A. Statistical Channel Power Profile}

Let $P(F)$ and $G(F)$ denote the Fourier transforms of $p(t)$ and $g(t)$, respectively. Let $\boldsymbol{H}=[H(1), H(2), \ldots, H(N)]^{T}$ denote the frequency-domain sub-carrier channel gains of the equivalent correlated channel tap vector $\boldsymbol{h}$. Then, we have the following proposition:

Proposition 1: If $|P(F)| \geq 0$ only for $|F| \leq K F_{s}$ ( $K$ is an integer and $F_{s}=\frac{1}{T_{s}}$ ) and $E\left\{g_{l} g_{l^{\prime}}^{*}\right\}=0$ for $l \neq l^{\prime}$, then the average channel power gain on the $n$th sub-carrier, denoted as $C_{n, n}$, is

$$
\begin{aligned}
& C_{n, n}=F_{s}^{2} \sum_{k_{1}=-K}^{K}\left|P\left(\left(\frac{n}{N}-k_{1}\right) F_{s}\right)\right|^{2}\left(\sum_{l=1}^{L} E\left[\left|g_{l}\right|^{2}\right]\right) \\
& +2 F_{s}^{2} \sum_{k_{1}=-K}^{K-1} \sum_{k_{2}=1}^{K-k_{1}}\left|P\left(\left(\frac{n}{N}-k_{1}\right) F_{s}\right)\right|\left|P\left(\left(\frac{n}{N}-k_{1}-k_{2}\right) F_{s}\right)\right| \\
& \times\left\{\sum_{l=1}^{L} E\left[\left|g_{l}\right|^{2} \cos \left(2 \pi k_{2}\left(\tau+t_{l}\right) F_{s}-\theta_{1}+\theta_{2}\right)\right]\right\},
\end{aligned}
$$

where $\theta_{1}$ is the phase of $P\left(\left(\frac{n}{N}-k_{1}\right) F_{s}\right)$ and $\theta_{2}$ is the phase of $P\left(\left(\frac{n}{N}-k_{1}-k_{2}\right) F_{s}\right)$. Note that if $p(t)$ is symmetric as typically used in practice, $\theta_{1}=\theta_{2}=0$.

Proof: The proof is given in Appendix I.

If $t_{l}=(l-1) T_{s},(9)$ becomes

$$
\begin{aligned}
& C_{n, n}=\left(\sum_{l=1}^{L} E\left(\left|g_{l}\right|^{2}\right)\right) F_{s}^{2}\left\{\sum_{k_{1}=-K}^{K}\left|P\left(\left(\frac{n}{N}-k_{1}\right) F_{s}\right)\right|^{2}\right. \\
& +2 \sum_{k_{1}=-K}^{K-1} \sum_{k_{2}=1}^{K-k_{1}}\left|P\left(\left(\frac{n}{N}-k_{1}\right) F_{s}\right)\right|\left|P\left(\left(\frac{n}{N}-k_{1}-k_{2}\right) F_{s}\right)\right| \\
& \left.\times \cos \left(2 \pi k_{2} \tau F_{s}-\theta_{1}+\theta_{2}\right)\right\} .
\end{aligned}
$$

It can be seen from (10) that the average power does not change with the exact value of $g_{l}$ and only varies with the total energy of all the taps, i.e., $\sum_{l=1}^{L} E\left(\left|g_{l}\right|^{2}\right)$.

The early-late gate synchronizer is a simple and practical method that is widely used for symbol synchronization and 


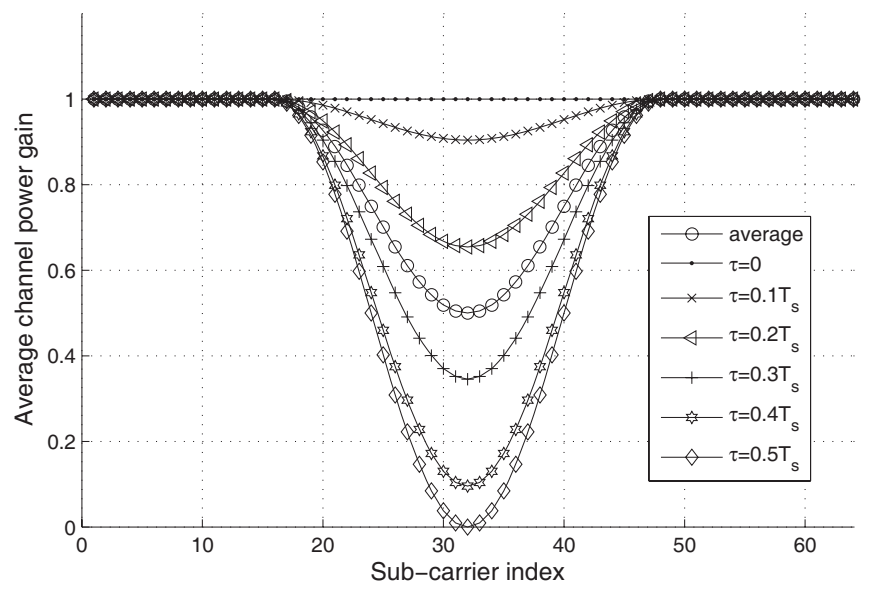

Fig. 1. Average channel power gain profile on the sub-carriers for the equivalent channel.

sampling at the receiver [6]. Define $\tau_{1}$ and $\tau_{2}$ as the arrival delays of Users 1 and 2, respectively. It is shown in [4] that the sampling time decided by the early-late gate synchronizer is about $\frac{\tau_{1}-\tau_{2}}{2}$ ahead of the ideal time for one signal and $\frac{\tau_{1}-\tau_{2}}{2}$ behind the ideal time for the other, i.e., $\tau=\frac{\tau_{1}-\tau_{2}}{2}$ for User 1 and $\tau=-\frac{\tau_{1}-\tau_{2}}{2}$ for User 2 . Without loss of generality, we now assume $0 \leq\left|\tau_{1}-\tau_{2}\right| \leq T_{s}$. In practical systems, $\tau$ is unknown at the receiver. Assume that $\tau$ is a random variable with probability density function (pdf) $f(\tau)$. Then, given $g_{l}$ and $t_{l}$, the average channel power profile is obtained by averaging (9) over $\tau$ as follows

$$
\bar{C}_{n, n}=\int_{-\infty}^{\infty} C_{n, n} f(\tau) d \tau
$$

Note that if $t_{l}$ is not fixed, we have to average (11) over $t_{l}$ to obtain the average power profile.

\section{Special Case Revisited:}

If the pulse shaping filter is the raised cosine function given in (4), then

$P(F)=\left\{\begin{array}{cc}T_{s}, & 0 \leq|F|<\frac{(1-\alpha)}{2 T_{s}} \\ \frac{T_{s}}{2}\left[1+\cos \left(\frac{\pi\left(|F| 2 T_{s}-(1-\alpha)\right)}{2 \alpha}\right)\right], & \frac{(1-\alpha)}{2 T_{s}} \leq|F|<\frac{(1+\alpha)}{2 T_{s}} \\ 0, & |F| \geq \frac{(1+\alpha)}{2 T_{s}}\end{array}\right.$

and $K=1$ in the Proposition 1 , and hence the average channel power gain on the $n$th sub-carrier can be easily calculated from (9). For $t_{l}=(l-1) T_{s}$, the numerical results of the average channel power gain obtained from (10) are shown in Fig. 1 with the assumption of $\tau$ being uniformly distributed over $\left[0, \frac{T_{s}}{2}\right]$ and $N=64$. From Fig. 1, we can see that the larger the timing synchronization error is, the smaller is the lowest channel power gain. Thus, larger timing synchronization errors cause more serious power unbalance among all the sub-carriers. Note that if a long pulse shape truncation length is used, it can be well approximated by the untruncated pulse for which (9) yields an easy computation of the channel power gains. For the equivalent channel vector given in (5) which only considers the first-order side lobes of the pulse shape (i.e., a short truncation length), the average power profile with $t_{l}=(l-1) T_{s}$ can be obtained from (10) by evaluating the Fourier transform of the time-domain truncated pulse shaping function. However, this involves the convolution of the raised cosine function with the sinc function, which is very complicated to evaluate. Thus, we analyze the power profile from the time-domain equivalent channel vector. From (5), the average channel power gain on the $n$th sub-carrier is given by

$$
\begin{aligned}
C_{n, n} & =\sum_{k=1}^{L} \sum_{m=1}^{L} R_{m, k} V_{n, m} V_{n, k}^{*} \\
& =\sum_{m=1}^{L} R_{m, m}+\sum_{k-m \neq 0} R_{m, k} e^{\frac{j 2 \pi(n-1)(k-m)}{N}},
\end{aligned}
$$

where $V_{n, m}=e^{-\frac{j 2 \pi(n-1)(m-1)}{N}}, R_{m, k}$ is the $(m, k)$-th element of $\boldsymbol{R}$, and $\boldsymbol{R}=E\left(\boldsymbol{h} \boldsymbol{h}^{H}\right)=\boldsymbol{P} E\left(\boldsymbol{g} \boldsymbol{g}^{H}\right) \boldsymbol{P}$. Then, we have the following proposition:

Proposition 2: The average power gain profile for the equivalent channel vector given in (5) for $0<\tau<T_{s}$ and $-T_{s}<\tau<0$ are respectively given as

$$
\begin{aligned}
& C_{n, n}=\left(\sum_{l=1}^{L} E\left(\left|g_{l}\right|^{2}\right)\right)\left\{\sum_{l^{\prime}=0}^{3} p_{l^{\prime}}^{2}+2 \cos \left(\frac{2 \pi(n-1)}{N}\right)\right. \\
& \times\left(p_{0} p_{1}+p_{1} p_{2}+p_{3} p_{0}\right)+2 \cos \left(\frac{4 \pi(n-1)}{N}\right)\left(p_{0} p_{2}+p_{3} p_{1}\right) \\
& \left.+2 \cos \left(\frac{6 \pi(n-1)}{N}\right)\left(p_{3} p_{2}\right)\right\}
\end{aligned}
$$

and

$$
\begin{aligned}
& C_{n, n}^{\prime}=\left(\sum_{l=1}^{L} E\left(\left|g_{l}\right|^{2}\right)\right)\left\{\sum_{l^{\prime}=0}^{3}{p^{\prime}}_{l^{\prime}}^{2}+2 \cos \left(\frac{2 \pi(n-1)}{N}\right)\right. \\
& \times\left(\sum_{l^{\prime}=0}^{2} p_{l^{\prime}}^{\prime} p^{\prime}{ }_{l^{\prime}+1}\right)+2 \cos \left(\frac{4 \pi(n-1)}{N}\right)\left({p^{\prime}}_{0} p^{\prime}{ }_{2}+p^{\prime}{ }_{3} p^{\prime}{ }_{1}\right) \\
& \left.+2 \cos \left(\frac{6 \pi(n-1)}{N}\right)\left(p^{\prime}{ }_{3} p^{\prime}{ }_{0}\right)\right\} .
\end{aligned}
$$

Proof: See Appendix II.

Regarding the above derivation, the following two remarks are in order:

- We have assumed that the timing error is $0<\left|\tau_{1}-\tau_{2}\right|<$ $T_{s}$. When the timing error is $D=d T+\tau_{1}-\tau_{2}$, where $d$ is an integer and $0<\tau_{1}-\tau_{2}<T_{s}$, the frequency-domainbased results in (9) and (10) still hold. The time-domainbased approach for a short pulse shape truncation length can also be applied with appropriate modifications.

- When there are more than two users, our derivation for the channel power gain profile given a specific timing error $\tau$ still holds. However, the value of $\tau$ may depend on the exact synchronization algorithm used in the system and the arrival delays of different users.

In Figs. 2 and 3, (14) and (15) are evaluated numerically for $N=64$ with the assumption of $\tau$ being uniformly distributed over $\left[0, \frac{T_{s}}{2}\right]$ and $\left[-\frac{T_{s}}{2}, 0\right]$, respectively. We observe that the truncated pulse shaping function results in a slightly larger imbalance of channel power gains across the subcarriers compared with the non-truncated case shown in Fig. 1. 


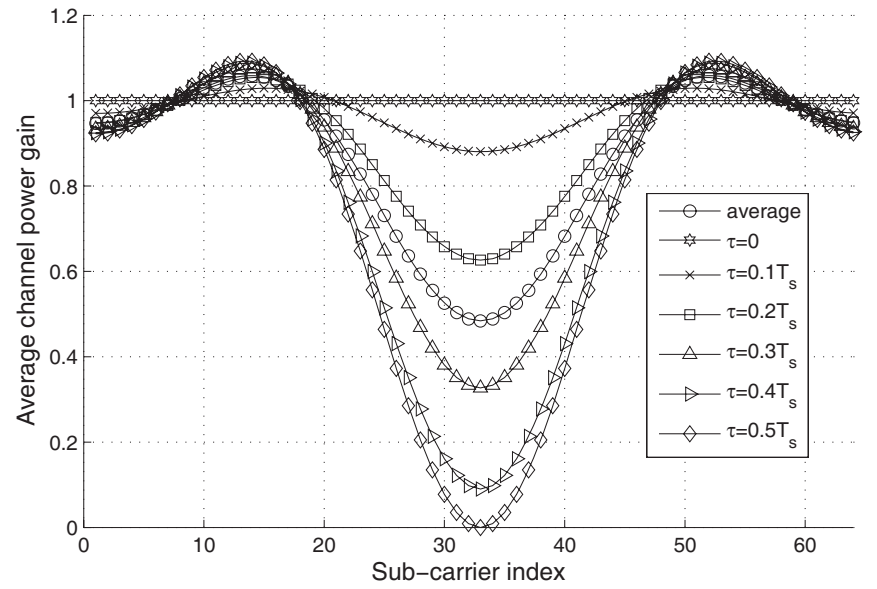

Fig. 2. Average channel power gain profile on the sub-carriers for the equivalent channel with truncated pulse shape function and $0<\tau<\frac{T_{s}}{2}$.

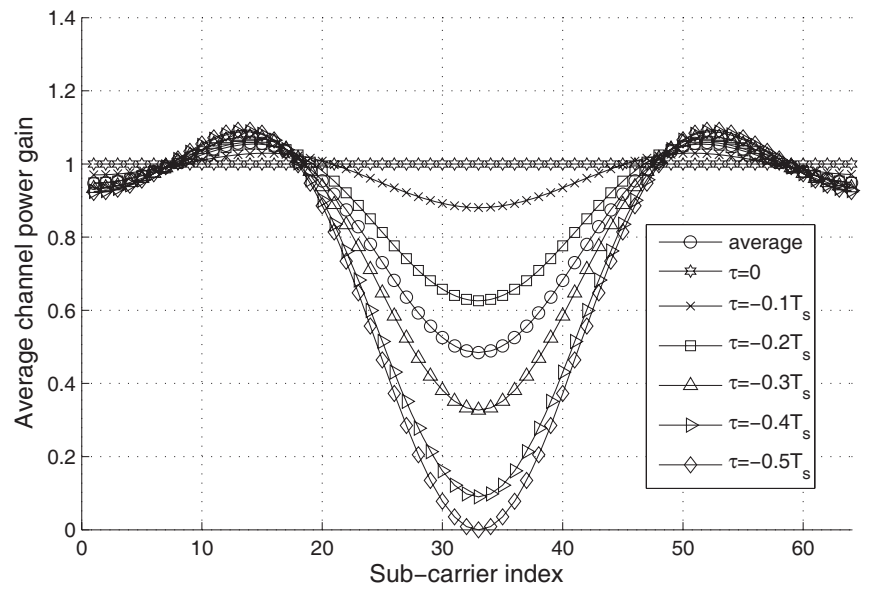

Fig. 3. Average channel power gain profile on the sub-carriers for the equivalent channel with truncated pulse shape function and $-\frac{T_{s}}{2}<\tau<0$.

\section{B. Statistical Bit Loading Algorithm}

In the previous subsection, the statistical channel power gain profile was derived. In this subsection, we will present a statistical bit loading algorithm to fully exploit it. A bit loading scheme, typically used in multicarrier wireline systems, assigns a high data rate to sub-carriers with high SNR and a low data rate to sub-carriers with low SNR [7]. In wireless systems, it is difficult to obtain the instantaneous channel information at the transmitter for fast time-varying channels and thus it is infeasible to adopt an adaptive bit loading algorithm. However, as shown in Fig. 1, the average channel power gain profile has a very special structure. Although we do not know the instantaneous channel information, it can be seen that there are some sub-carriers which have less average channel power gain than the other sub-carriers. With this average power profile, we propose a statistical bit loading scheme. In [8], a statistical bit loading scheme was proposed to maximize the overall spectral efficiency and minimize the transmission power requirement while maintaining the target BER level. However, in this paper, our objective is to develop a transmission scheme where all sub-carriers have the same smallest bit error rate (BER) given total power and total data rate constraints.
1) Approximate BER Expression for Each Sub-carrier : Here, we will derive the BER performance for each subcarrier, where a maximum likehood (ML) receiver is used at the base station as an interference cancelation detector. With ML receiver, the diversity order of each user in the multiuser case in SDMA is the same as the diversity order in the single-user case, and for analytical tractability, we neglect interference among users in the BER expression used in our bit loading algorithm.

Since the channel response on each sub-carrier is a flatRayleigh-fading channel with variance $C_{n, n}$, it is equivalent to derive the BER performance for a single user using OSTBC with $N_{t}$ transmit antennas and $N_{r}$ receive antennas under Rayleigh flat-fading channels. A MIMO fading channel with OSTBC transmission can be transformed into an equivalent Gaussian SISO channel [9]. Then, on the $n$th sub-carrier, the pdf and the moment generation function (MGF) of the instantaneous signal-noise-ratio (SNR) $\gamma_{n}$ of the equivalent SISO model are given by

$$
p_{\gamma_{n}}\left(\gamma_{n}\right)=\frac{\gamma_{n}^{N_{t} N_{r}-1}}{\Gamma\left(N_{t} N_{r}\right) \bar{\gamma}_{n}^{N_{t} N_{r}}} e^{-\frac{\gamma}{\gamma_{n}}}
$$

and

$$
\phi_{\gamma_{n}}(s) \triangleq \int_{0}^{\infty} e^{-s \gamma_{n}} p_{\gamma_{n}}\left(\gamma_{n}\right) d \gamma_{n}=\left(1+s \bar{\gamma}_{n}\right)^{-N_{t} N_{r}},
$$

respectively, where $\bar{\gamma}_{n} \triangleq \frac{S_{n} C_{n, n}}{N_{t} \xi N_{0}}$. The quantities $S_{n}, \xi$ and $N_{0}$ are the total transmit power (over all transmit antennas) on the $n$th sub-carrier, the OSTBC rate and the noise variance, respectively. The authors in [9] also gave the exact symbol error probability of OSTBC for flat-Rayleigh-fading channels. However, the expression is too complicated to use in the bit loading scheme. Here, we will derive an approximation BER expression.

A simple approximate BER expression for square MQAM ${ }^{1}$ with Gray bit mapping in additive white Gaussian noise (AWGN) channels is given in [10] as

$$
P_{e_{n} \mid \gamma_{n}} \cong 0.2 e^{\frac{-1.6 \gamma_{n}}{M-1}} \text {. }
$$

Let $2^{k_{n}}$ denote the constellation size on the $n$th sub-carrier. Then, the BER on the $n$th sub-carrier is given by

$$
\begin{aligned}
& P_{e_{n}}=\int_{0}^{\infty} P_{e_{n} \mid \gamma_{n}} p_{\gamma_{n}}\left(\gamma_{n}\right) d_{\gamma_{n}}=\int_{0}^{\infty} 0.2 e^{\frac{-1.6 \gamma_{n}}{2^{k_{n}-1}}} p_{\gamma_{n}}\left(\gamma_{n}\right) d_{\gamma_{n}} \\
& =0.2 \phi_{\gamma_{n}}\left(\frac{1.6}{2^{k_{n}}-1}\right)=\frac{0.2}{\left(1+\frac{1.6}{2^{k_{n}}-1} \frac{S_{n} C_{n, n}}{N_{t} \xi N_{0}}\right)^{N_{t} N_{r}}}
\end{aligned}
$$

2) Statistical Bit Loading Algorithm: Our statistical bit loading algorithm solves the following optimization problem:

$$
\begin{array}{ll}
\underset{R_{n}, S_{n}}{\arg \min } & P_{e}=\frac{0.2}{\left(1+\frac{1.6}{2^{k_{n}-1}} \frac{S_{n} C_{n, n}}{N_{t} \xi N_{0}}\right)^{N_{t} N_{r}}}=P_{e_{n}}, \forall n \\
\text { subject to } & \left\{\begin{array}{l}
(a) \sum_{n=1}^{N} k_{n}=R_{T} \\
\text { (b) } \sum_{n=1}^{N} S_{n}=S_{T} \\
(c) P_{e_{n}} \text { is the same for all } n=1, \ldots, N .
\end{array}\right.
\end{array}
$$

\footnotetext{
${ }^{1}$ For analytical tractability in the bit loading algorithm, we use this expression for non-square MQAM as well.
} 
From (20), we know that minimizing $P_{e}$ is equivalent to maximizing

$$
y=\frac{S_{n} C_{n, n}}{2^{k_{n}}-1} .
$$

From (21), we obtain

$$
S_{n}=\frac{y\left(2^{k_{n}}-1\right)}{C_{n, n}} .
$$

Substituting (22) into Constraint (b) of (20), we obtain

$$
y=\frac{S_{T}}{\sum_{n=1}^{N} \frac{2^{k_{n}-1}}{C_{n, n}}}=\frac{S_{T}}{\sum_{n=1}^{N} \frac{2^{k_{n}}}{C_{n, n}}-\sum_{n=1}^{N} \frac{1}{C_{n, n}}} .
$$

Thus, maximizing $y$ is equivalent to minimizing $\sum_{n=1}^{N} \frac{2^{k_{n}}}{C_{n, n}}$. Performing the Lagrange optimization under Constraint $(a)$ of (20), we obtain

$$
k_{n}=\frac{R_{T}}{N}-\frac{1}{N} \log _{2}\left(\prod_{m=1}^{N}\left(C_{m, m}\right)\right)+\log _{2}\left(C_{n, n}\right) .
$$

We summarize the proposed statistical bit loading algorithm in the following five steps:

- Step 1: Select active sub-carriers

$$
k_{n}=\frac{R_{T}}{|I|}-\frac{1}{|I|} \log _{2}\left(\prod_{m=1}^{|I|}\left(C_{m, m}\right)\right)+\log _{2}\left(C_{n, n}\right) .
$$

Initially, $I=\{1,2, \ldots, N\}$ where $|I|$ denotes the size of the set $I$. If $k_{n}<0$, then remove sub-carrier $n$ from $I$. This is done iteratively until all rates of the remaining sub-carriers are positive.

- Step 2: $k_{n}$ is quantized to $Q_{n}$ as

$$
Q_{n}=\left\{\begin{array}{ll}
R_{\max } & : k_{n}>=R_{\max }-0.5 \\
\text { Floor }\left(k_{n}+0.5\right) & : 0.5<k_{n}<R_{\max }-0.5 \\
0 & : k_{n}<0.5,
\end{array},\right.
$$

where $R_{\max }$ is the predefined maximum signal constellation size on a sub-carrier.

- Step 3: If $\sum_{n \in I} Q_{n},<(>) R_{T}$, the rate of the channel with the largest (smallest) $\Delta k_{n}=k_{n}-Q_{n}$, is incremented (decremented). The initial bit allocation stops if $\sum_{n \in I} Q_{n}=R_{T}$.

- Step 4: If the number of sub-carriers with $Q_{n}=1$ is odd, to adjust it to be even, we reduce the constellation size of the sub-carrier with the smallest average channel power gain among those with the largest constellation size by ' 1 ' bit. Based on the results from Step 3, adjust the bit allocations on the sub-carriers with $Q_{n}=1$ such that half of these sub-carriers with the higher (lower) channel power gain profile are assigned $Q_{n}=2\left(Q_{n}=0\right)$ so that $\sum_{n \in I} Q_{n}=R_{T}$.

- Step 5: Transmit power is assigned to the sub-carriers recorded in the set $I$ such that all sub-carriers achieve the same error probability, i.e.,

$$
S_{n}=\frac{\frac{S_{T}\left(2^{Q_{n}}-1\right)}{C_{n, n}}}{\sum_{l \in I} \frac{2^{Q_{l}-1}}{C_{l, l}}}
$$

where $S_{T}$ is the fixed total transmit power.
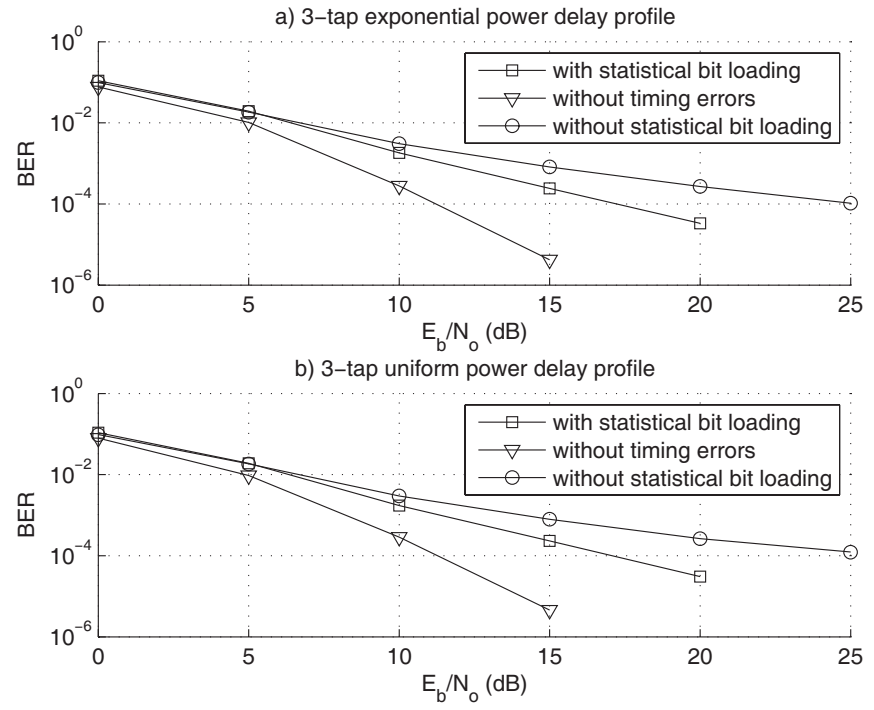

Fig. 4. BER performance comparison with/without statistical bit loading for 3-tap Rayleigh fading channels with exponential/uniform power delay profile.

\section{Simulation Results}

In our simulations, the channel statistics between different transmit antennas and different receive antennas are assumed to be the same, each of which is modeled as the following four types of channels:

- 3-tap Rayleigh fading channel with an exponential power delay profile (the tap variance decays at $3 \mathrm{~dB} / \mathrm{tap}$ ) and $t_{l}=(l-1) T_{s}$.

- 3-tap Rayleigh fading channel with a uniform power delay profile and $t_{l}=(l-1) T_{s}$.

- 7-tap Rayleigh fading channel with an exponential power delay profile (the tap variance decays at $3 \mathrm{~dB} / \mathrm{tap}$ ) and $t_{l}=(l-1) T_{s}$.

- 3-tap Rician fading channel with an exponential power delay profile (the tap variance decays at $3 \mathrm{~dB} / \mathrm{tap}$ ) and $t_{l}=(l-1) T_{s}$. The first tap is Rice distributed, where the real part has a Gaussian distribution of mean $\sqrt{0.4}$ and the imaginary part has a Gaussian distribution of zero mean. Since the complicated BER expression for Rician fading channel introduces an analytical obstacle in the statistical bit loading algorithm, we use the algorithm derived based on the Rayleigh fading channel in Section III for the Rician fading channel as well. This will reflect performance sensitivity of the proposed scheme to channel model mismatches.

The equivalent channels impaired by the timing synchronization errors are assumed to be perfectly known at the receiver ${ }^{2}$. Note that the transmitters do not have knowledge of the instantaneous channel gains. The difference of the arrival delays from Users 1 and 2 are assumed to be uniformly distributed over $\left[-T_{s}, T_{s}\right]$. We consider two transmit antennas at each user and two receive antennas at the base station. In both cases, there are $N=64$ sub-carriers. The ML detector is implemented on each sub-carrier to decode the source information.

\footnotetext{
${ }^{2}$ In practice, they are obtained using channel estimation techniques.
} 


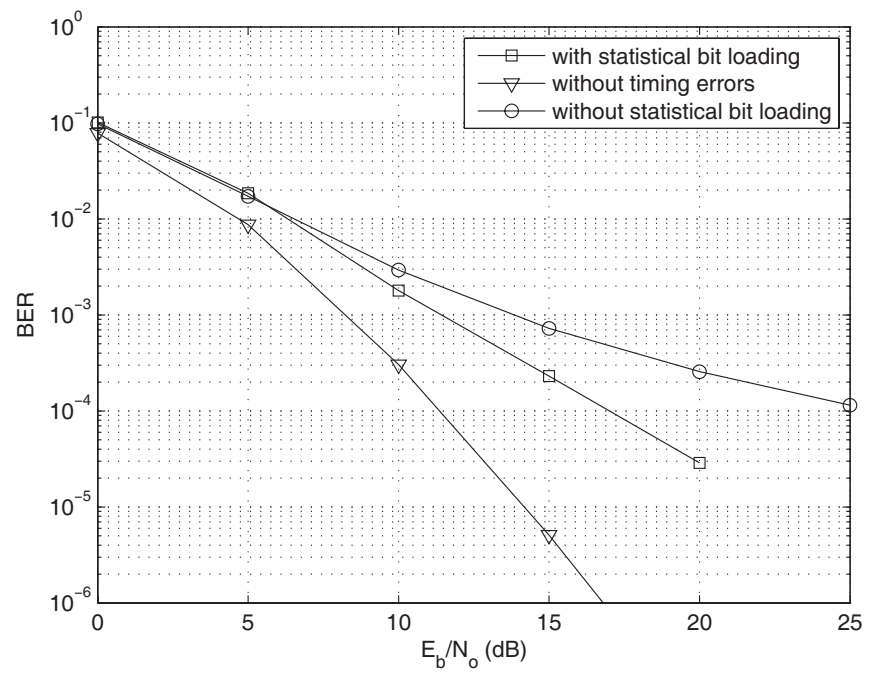

Fig. 5. BER performance comparison with/without statistical bit loading for 7-tap Rayleigh fading channels with exponential power delay profile.

Figs. 4, 5, and 6 present the BER performance comparison of our proposed scheme and the conventional scheme without statistical bit loading assuming two transmit antennas for different channel types. Also, the performance without synchronization errors is given as a benchmark, in which the average channel power gains on all sub-carriers are the same and no statistical bit loading scheme is needed. The results show that the proposed scheme is not sensitive to the channel type. Our proposed algorithm improves the robustness to timing synchronization errors, yielding an SNR advantage of around $7.5 \mathrm{~dB}$ at $\mathrm{BER}=10^{-4}$ in the channels considered.

\section{CONCLUSIONS}

A novel STBC-OFDM transmission scheme robust to timing synchronization errors over frequency-selective multipleaccess channels is proposed in this paper. The analysis of the statistical power gain profile of the correlated equivalent channel model shows that the average channel power gains on some sub-carriers are always less than the others. A robust statistical bit loading algorithm is proposed to exploit this average channel power profile resulting in an appreciable performance improvement. This algorithm is appealing to systems where link adaption based on instantaneous channel information is infeasible or undesirable.

\section{APPENDIX A}

\section{ProOf of PRoposition 1}

From (2), we obtain

$$
H_{a}(F)=P(F) G(F) e^{-j 2 \pi F \tau} .
$$

Let $h(n)=h_{a}\left(n T_{s}\right)$. Then, its frequency domain representation is given by [11]

$$
H(f)=F_{s} \sum_{k=-\infty}^{\infty} H_{a}\left[(f-k) F_{s}\right],
$$

where $f=\frac{F}{F_{s}}$, representing the relationship between the frequency variables $F$ and $f$ of the corresponding analog and discrete-time signals, respectively.

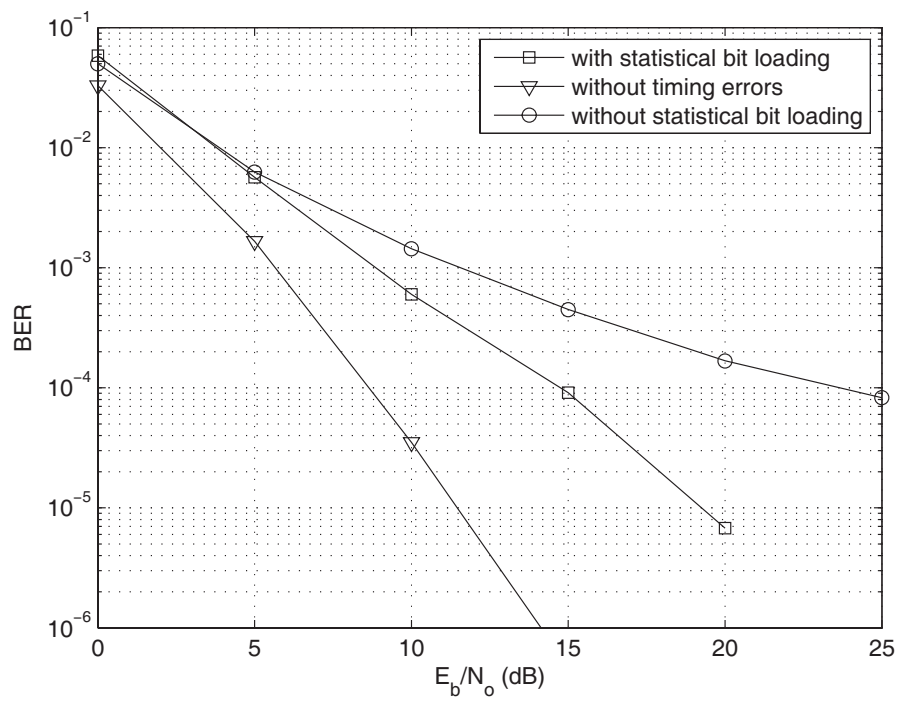

Fig. 6. BER performance comparison with/without statistical bit loading for 3-tap Rician channels with exponential power delay profile

If $|P(F)| \geq 0$ only for $|F| \leq K F_{s}$, then

$H(f)=F_{s} \sum_{k=-K}^{K} P\left((f-k) F_{s}\right) G\left((f-k) F_{s}\right) e^{-j 2 \pi(f-k) F_{s} \tau}$.

Since there are $N$ sub-carriers, the frequency response on the $n$th sub-carrier is

$H\left(\frac{n}{N}\right)=F_{s} \sum_{k=-K}^{K} P\left(\left(\frac{n}{N}-k\right) F_{s}\right) G\left(\left(\frac{n}{N}-k\right) F_{s}\right) e^{-j 2 \pi\left(\frac{n}{N}-k\right) F_{s} \tau}$.

Let $x(k)=P\left(\left(\frac{n}{N}-k\right) F_{s}\right) G\left(\left(\frac{n}{N}-k\right) F_{s}\right) e^{-j 2 \pi\left(\frac{n}{N}-k\right) F_{s} \tau}$. Then, the average channel power gain on the $n$th sub-carrier is given as

$$
\begin{aligned}
C_{n, n} & =E\left(\left|H\left(\frac{n}{N}\right)\right|^{2}\right) \\
& =F_{s}^{2} \sum_{k_{1}=-K}^{K-1} \sum_{k_{2}=1}^{K-k_{1}} 2 \Re\left\{E\left[x\left(k_{1}\right) x^{*}\left(k_{1}+k_{2}\right)\right]\right\} \\
& +F_{s}^{2} \sum_{k_{1}=-K}^{K} E\left(\left|x\left(k_{1}\right)\right|^{2}\right) .
\end{aligned}
$$

Let $g(t)=\sum_{l=1}^{L} g_{l} \delta\left(t-t_{l}\right)$. Then

$$
G(F)=\int_{-\infty}^{\infty} g(t) e^{-j 2 \pi F t} d t=\sum_{l=1}^{L} g_{l} e^{-j 2 \pi F t_{l}} .
$$

Since $E\left\{g_{l} g_{l^{\prime}}^{*}\right\}=0$ for $l \neq l^{\prime}$, we have

$$
\begin{aligned}
& E\left[G\left(\left(\frac{n}{N}-k_{1}\right) F_{s}\right) G^{*}\left(\left(\frac{n}{N}-k_{1}-k_{2}\right) F_{s}\right)\right] \\
& =E\left[\sum_{l_{1}=1}^{L} g_{l_{1}} e^{-j 2 \pi\left(\frac{n}{N}-k_{1}\right) F_{s} t_{l_{1}}} \sum_{l_{2}=1}^{L} g_{l_{2}}^{*} e^{j 2 \pi\left(\frac{n}{N}-k_{1}-k_{2}\right) F_{s} t_{l_{2}}}\right] \\
& =E\left[\sum_{l=1}^{L}\left|g_{l}\right|^{2} e^{-j 2 \pi k_{2} F_{s} t_{l}}\right]=\sum_{l=1}^{L} E\left[\left|g_{l}\right|^{2} e^{-j 2 \pi k_{2} F_{s} t_{l}}\right] .
\end{aligned}
$$


Therefore,

$$
\begin{aligned}
& E\left[x\left(k_{1}\right) x^{*}\left(k_{1}+k_{2}\right)\right] \\
& =\left|P\left(\left(\frac{n}{N}-k_{1}\right) F_{s}\right)\right|\left|P\left(\left(\frac{n}{N}-k_{1}-k_{2}\right) F_{s}\right)\right| e^{j \theta_{1}} e^{-j \theta_{2}} \\
& \times\left\{\sum_{l=1}^{L} E\left[\left|g_{l}\right|^{2} e^{-j 2 \pi k_{2}\left(\tau+t_{l}\right) F_{s}}\right]\right\},
\end{aligned}
$$

where $\theta_{1}$ is the phase of $P\left(\left(\frac{n}{N}-k_{1}\right) F_{s}\right)$ and $\theta_{2}$ is the phase of $P\left(\left(\frac{n}{N}-k_{1}-k_{2}\right) F_{s}\right)$. Then,

$$
\begin{aligned}
& \Re\left\{E\left[x\left(k_{1}\right) x^{*}\left(k_{1}+k_{2}\right)\right]\right\} \\
& =\left|P\left(\left(\frac{n}{N}-k_{1}\right) F_{s}\right)\right|\left|P\left(\left(\frac{n}{N}-k_{1}-k_{2}\right) F_{s}\right)\right| \times \\
& \left\{\sum_{l=1}^{L} E\left[\left|g_{l}\right|^{2} \cos \left(2 \pi k_{2}\left(\tau+t_{l}\right) F_{s}-\theta_{1}+\theta_{2}\right)\right]\right\} .
\end{aligned}
$$

Substituting (36) into (32) gives (9).

\section{APPENDIX B}

Proof OF Proposition 2

Denote $p=|k-m| \geq 1$. From (13), we have

$C_{n, n}=\sum_{m=1}^{L} R_{m, m}$
$+\sum_{p=1}^{L-1}\left[\sum_{m=1}^{L-p} R_{m, m+p} e^{\frac{j 2 \pi(n-1) p}{N}}+\sum_{m=1}^{L-p} R_{m+p, m} e^{\frac{j 2 \pi(n-1)(-p)}{N}}\right]$.

Since $\boldsymbol{R}$ is a Hermitian matrix, $R_{m+p, m}=R_{m, m+p}^{*}$. We have

$$
\begin{aligned}
& \sum_{m=1}^{L-p} R_{m, m+p} e^{\frac{j 2 \pi(n-1) p}{N}}+\sum_{m=1}^{L-p} R_{m+p, m} e^{\frac{j 2 \pi(n-1)(-p)}{N}} \\
& =\sum_{m=1}^{L-p}\left[2 \Re\left(R_{m, m+p}\right) \cos \left(\frac{2 \pi(n-1) p}{N}\right)\right. \\
& \left.+2 \Im\left(R_{m, m+p}\right) \sin \left(\frac{2 \pi(n-1) p}{N}\right)\right]
\end{aligned}
$$

Thus, (37) becomes

$$
\begin{aligned}
& C_{n, n}=\sum_{m=1}^{L} R_{m, m}+2 \sum_{p=1}^{L-1} \cos \left(\frac{2 \pi(n-1) p}{N}\right) \sum_{m=1}^{L-p} \Re\left(R_{m, m+p}\right) \\
& +2 \sum_{p=1}^{L-1} \sin \left(\frac{2 \pi(n-1) p}{N}\right) \sum_{m=1}^{L-p} \Im\left(R_{m, m+p}\right) .
\end{aligned}
$$

If all the elements of $\boldsymbol{R}$ are real, e.g., a channel with uncorrelated zero-mean channel taps, then (39) becomes

$C_{n, n}=\sum_{m=1}^{L} R_{m, m}+2 \sum_{p=1}^{L-1} \cos \left(\frac{2 \pi(n-1) p}{N}\right) \sum_{m=1}^{L-p} \Re\left(R_{m, m+p}\right)$.

Since $\boldsymbol{R}=\boldsymbol{P R}_{\boldsymbol{g}} \boldsymbol{P}^{H}$ and $\boldsymbol{R}_{\boldsymbol{g}}=$ $\operatorname{diag}\left\{E\left(\left|g_{1}\right|^{2}\right), E\left(\left|g_{2}\right|^{2}\right), \ldots, E\left(\left|g_{L}\right|^{2}\right)\right\}$ with $P_{i, j}$ denoting the $(i, j)$-th element of $\boldsymbol{P}$, we have

$$
R_{m, k}=\sum_{l=1}^{L} \sum_{r=1}^{L} P_{m, r} R_{g}(r, l) P_{k, l}^{*}=\sum_{l=1}^{L} P_{m, l} E\left(\left|g_{l}\right|^{2}\right) P_{k, l}^{*} .
$$

Thus,

$$
\sum_{m=1}^{M} R_{m, m}=\sum_{l=1}^{L} E\left(\left|g_{l}\right|^{2}\right) \sum_{m=1}^{L}\left|P_{m, l}\right|^{2} .
$$

From (6), we get

$$
\sum_{m=1}^{L}\left|P_{m, l}\right|^{2}=p_{0}^{2}+p_{1}^{2}+p_{2}^{2}+p_{3}^{2}, l=1,2, \ldots, L,
$$

and (42) becomes

$$
\sum_{m=1}^{L} R_{m, m}=\left(p_{0}^{2}+p_{1}^{2}+p_{2}^{2}+p_{3}^{2}\right) \sum_{l=1}^{L} E\left(\left|g_{l}\right|^{2}\right) .
$$

From (41), we get

$$
\sum_{m=1}^{L-p} \Re\left(R_{m, m+p}\right)=\sum_{l=1}^{L} E\left(\left|g_{l}\right|^{2}\right) \sum_{m=1}^{L-p} P_{m, l} P_{m+p, l}^{*} .
$$

From (6), we know that

$$
\sum_{m=1}^{L-p} P_{m, l} P_{m+p, l}^{*}=\left\{\begin{array}{crl}
p_{0} p_{1}+p_{1} p_{2}+p_{3} p_{0} & , p=1 \\
p_{0} p_{2}+p_{3} p_{1} & , p=2 \\
p_{3} p_{2} & , p=3 \\
0 & , \text { otherwise }
\end{array}\right.
$$

Thus, (40) becomes (14). Similarly, we can obtain $C_{n, n}$ for $-T_{s}<\tau<0$ from (8) as in (15).

\section{REFERENCES}

[1] N. Al-Dhahir, "Overview and comparison of equalization schemes for space-time-coded signals with application to EDGE," IEEE Trans. Signal Processing, vol. 50, pp. 2477-2488, Oct. 2002.

[2] R. L. Choi, M. T. Ivrlac, R. D. Murch, and W. Utschick, "On strategies of multiuser MIMO transmit signal processing," IEEE Trans. Wireless Commun., vol. 3, no. 6, pp. 1936-1941, Nov. 2004.

[3] A. F. Naguib, N. Seshadri, and A. R. Calderbank, "Applications of space-time block codes and interference suppression for high capacity and high data rate wireless systems," in Proc. 32nd Asilomar Conf. Signals, Systems, and Computers, Nov. 1998, vol. 2, pp. 1803-1810.

[4] Y. Mei, Y. Hua, A. Swami, and B. Daneshrad, "Combating synchronization errors in cooperative relays," in Proc. IEEE ICASSP, Mar. 2005, vol. 3, pp. 18-23.

[5] V. Tarokh, H. Jafarkhani, and A. R. Calderbank, "Space-time block coding for wireless communications: performance results," IEEE J. Select. Areas Commun., vol. 17, no. 3, pp. 451-460, 1999.

[6] J. G. Proakis, Digital Communications. McGraw-Hill, 4th edition, 2001.

[7] R. F. H. Fisher and J. B. Huber, "A new loading algorithm for discrete multitone transmission," in Proc. IEEE Globecom, Nov. 1996, vol. 1, pp. 724-728.

[8] Z. Song, K. Zhang, and Y. Guan, "Joint bit-loading and power-allocation for OFDM systems based on statistical frequency-domain fading model," in Proc. IEEE VTC, Fall 2002, vol. 2, pp. 724-728.

[9] H. Shin and J. H. Lee, "Exact symbol error probability of orthogonal space-time block codes," in Proc. IEEE Global Telecomm. Conf., Nov. 2002, vol. 2, pp. 1197-1201.

[10] S. T. Chung and A. J. Goldsmith, "Degrees of freedom in adaptive modulation: a unified view," IEEE Trans. Commun., vol. 49, pp. 15611571, Sept. 2001.

[11] J. G. Proakis and D. G. Manolakis, Digital Signal Processing: Principles, Algorithms, and Applications. Prentice Hall, 3rd edition, 1996. 